\section{What Are the Costs of Corneal Cross-linking for the Treatment of Progressive Keratoconus?}

\section{To the Editor:}

Although corneal cross-linking (CXL) for keratoconus is increasingly applied, there is limited evidence on the costs of this treatment. One cost-effectiveness study estimated the costs of the total CXL treatment for one simulated patient as $£ 928$ (\$1,392 U.S.). ${ }^{1}$ In our study, we assessed the costs of CXL in clinical practice, including preoperative eligibility assessments and 1-year follow-up.

All consecutive patients referred for keratoconus to our tertiary referral center between September 2012 and July 2013 were included. Surgical treatment was performed according to the Dresden protocol $\left(3 \mathrm{~mW} / \mathrm{cm}^{2}\right.$ irradiation for 30 minutes). Inclusion and exclusion criteria were described previously. ${ }^{2}$ All costs of preoperative consultation, surgical procedure, and postoperative costs during 1 year of follow-up were assessed from the health care perspective, following a microcosting approach in concordance with Dutch guidelines for health economic research. ${ }^{3}$ Equipment costs were based on purchase value, annual volume, and a 10-year depreciation. Costs of medication were obtained from the Dutch Healthcare Institution. ${ }^{4}$ All costs were converted to 2015 Euros and an exchange rate of 1.10 to U.S. dollars was applied.

A total of 43 patients (86 eyes) were included in this study. Twenty-eight eyes of 20 patients were scheduled for CXL treatment. The mean age of treated patients was 20.2 years and mean uncorrected and corrected visual acuity of treated eyes were 20/100 and 20/29, respectively, with a mean maximum keratometry value of 57.50 diopters. The mean total costs for one eye treated with CXL were $€ 1,754.06$ ( \pm 177.23 ) or $\$ 1,929.47$ ( \pm 194.95 ). The mean costs of the surgical CXL procedure itself were $€ 719.50$ ( \pm 18.82 ) of $\$ 791.45$ ( \pm 20.70 ) (Table A, available in the online version of this article). Personnel costs were the main driver, accountable for $88.1 \%$ of the total costs. Further cost categories, such as medication, diagnostic procedures, and the ultraviolet-A lamp, were accountable for $4.6 \%, 4.3 \%$, and 3\%, respectively (Figure 1).

Although personnel costs are the main expense in the CXL treatment, it should be taken into account that wages can diverge among organizations and countries. In our institution, the surgical procedure is performed by an optometrist, as are parts of the preoperative and postoperative consultation. The investment of medical specialist time is therefore reduced. If all care would be delivered by an ophthalmologist, the costs of the treatment itself would increase by $23.5 \%$ and the overall cost by $20.3 \%$ (calculations not shown). Accelerated CXL has been shown to produce similar results with shorter ultraviolet-A radiation times and thus shorter surgical procedure times. ${ }^{5} \mathrm{~A}$ decreased radiation time from 30 to 5 minutes

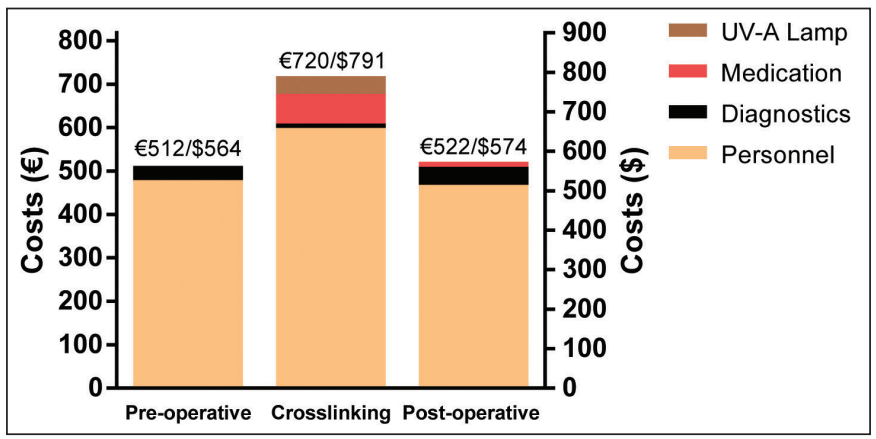

Figure 1. Costs of one eye treated with corneal cross-linking for progressive keratoconus during preoperative consultation, surgical cross-linking procedure, and 1-year postoperative follow-up. The total costs for one eye treated with cross-linking were $€ 1,754.06$ or $\$ 1,929.47$. UVA $=$ ultraviolet-A

would save $€ 141.09$ or $\$ 155.20$ (8.0\% of total costs and $19.6 \%$ of CXL treatment costs). The relatively small decrease is explained by the fact that the majority of costs are incurred during the preoperative consultations and postoperative follow-up period. More detailed insight is provided in Table A. This table can also be used to adjust wages and material costs to local situations.

These results provide insight in the financial side of CXL treatment and can be used in health economic evaluations, procedural pricing, and reimbursement negotiations.

\section{REFERENCES}

1. Salmon HA, Chalk D, Stein K, et al. Cost effectiveness of collagen crosslinking for progressive keratoconus in the UK NHS. Eye (Lond). 2015;29:1504-1511

2. Wisse RP, Godefrooij DA, Soeters N, et al. A multivariate analysis and statistical model for predicting visual acuity and keratometry one year after cross-linking for keratoconus. Am J Ophthalmol. 2014;157:519-525.

3. Oostenbrink JB, Bouwmans CAM, Koopmanschap MA, et al. Handleiding voor Kostenonderzoek: Methoden en Standaard Kostprijzen voor Economische Evaluaties in de Gezondheidszorg. Diemen: College voor Zorgverzekeringen; 2010.

4. Dutch Healthcare Institution. Information about drug costs [article in Dutch]. Available at: http://www.medicijnkosten.nl. Accessed: July 10, 2015

5. Hashemi H, Fotouhi A, Miraftab M, et al. Short-term comparison of accelerated and standard methods of corneal collagen crosslinking. J Cataract Refract Surg. 2015;41:533-540.

Daniel A. Godefrooij, MD Pepijn van Geuns, BSc G. Ardine de Wit, PhD

Robert P. L. Wisse, MD, PhD

Utrecht, the Netherlands

Supported by unrestricted grants from the Dr. F.P. Fischer Foundation ( $D G, R W$ ), facilitated by the Foundation Friends from the UMC Utrecht.

The authors have no financial or proprietary interest in the materials presented herein.

doi:10.3928/1081597X-20160318-01 


\section{TABLE A}

Costs of Cross-linking Treatment for Keratoconus Including Preoperative Assessment and Follow-up During 1 Year

\begin{tabular}{|c|c|c|c|}
\hline Parameter & Costs Per Unit $(€)$ & Units of Resource Use (Mean) & Mean Costs $(€)$ \\
\hline \multicolumn{4}{|l|}{ Preoperative costs } \\
\hline \multicolumn{4}{|l|}{ First outpatient visit $(20+20$ minutes $)$} \\
\hline Corneal specialist & 145.55/visit & 1.00 & 145.55 \\
\hline Optometrist & 112.87/visit & 1.00 & 112.87 \\
\hline \multicolumn{4}{|l|}{ Diagnostic procedures } \\
\hline Keratometry & 5.72/eye & 2.00 & 11.44 \\
\hline Endothelial cell count & 3.68/eye & 1.05 & 3.85 \\
\hline \multicolumn{4}{|l|}{ Progression control consultation (15 minutes) } \\
\hline Corneal specialist & 109.16/visit & 0.05 & 5.08 \\
\hline Optometrist & 84.66/visit & 0.42 & 35.44 \\
\hline Resident & 89.12/visit & 0.02 & 2.07 \\
\hline Nurse practitioner & 85.31/visit & 0.05 & 3.97 \\
\hline \multicolumn{4}{|l|}{ Diagnostic procedures } \\
\hline Keratometry & 5.72/visit & 1.07 & 6.12 \\
\hline Endothelial cell count & 3.68/eye & 0.09 & 0.34 \\
\hline \multicolumn{4}{|l|}{ Consultation by telephone (10 minutes) } \\
\hline Corneal specialist & 3.12/call & 0.12 & 2.27 \\
\hline Optometrist & 3.12/call & 0.12 & 0.36 \\
\hline Non-patient-related consultation & 11.32/patient & 0.33 & 3.69 \\
\hline No shows & 12.50/visit & 0.05 & 0.58 \\
\hline Costs per evaluated patient $(n=43)$ & & & $333.63 \pm 123.30$ \\
\hline Costs per treated eye $(n=28)$ & & & $512.36 \pm 52.45$ \\
\hline \multicolumn{4}{|l|}{ Cross-linking treatment } \\
\hline \multicolumn{4}{|l|}{ Procedural costs } \\
\hline Optometrist (105 minutes) & 592.57/visit & 1.00 & 592.57 \\
\hline Abrasion & 5.79/abrasion & 1.00 & 5.79 \\
\hline \multicolumn{4}{|l|}{ Materials } \\
\hline UVA lamp & 37.01/eye & 1.00 & 37.01 \\
\hline Pachymeter & 10.36/eye & 1.00 & 5.79 \\
\hline Bandage contact lens & 4.42/eye & 1.00 & 4.42 \\
\hline \multicolumn{4}{|l|}{ Medication } \\
\hline Riboflavin & 59.61/eye & 1.00 & 59.61 \\
\hline Hypotone riboflavin & 59.61/eye & 0.11 & 6.39 \\
\hline Preoperative eye drops & 2.77/eye & 1.00 & 2.77 \\
\hline No shows & 16.39/visit & 0.04 & 0.59 \\
\hline Costs of surgical cross-linking procedure $(n=28)$ & & & $719.50 \pm 18.82$ \\
\hline \multicolumn{4}{|l|}{ Postoperative costs } \\
\hline \multicolumn{4}{|l|}{ Medication } \\
\hline Postoperative eye drops & 11.78/eye & 1.00 & 11.78 \\
\hline Oral medication & 0.40/eye & 1.00 & 0.40 \\
\hline \multicolumn{4}{|l|}{ Outpatient visit ( $\leq 1$ week, 10 minutes) } \\
\hline Corneal specialist & 72.78/visit & 0.25 & 18.20 \\
\hline Optometrist & 56.44/visit & 0.64 & 36.28 \\
\hline Resident & 59.42/visit & 0.36 & 21.22 \\
\hline Nurse practitioner & $56.87 /$ visit & 0.07 & 4.06 \\
\hline \multicolumn{4}{|l|}{ Outpatient visit (> 1 week, 20 minutes) } \\
\hline Corneal specialist & 145.55/visit & 0.11 & 15.59 \\
\hline Optometrist & 112.87/visit & 2.64 & 298.30 \\
\hline Resident & 118.83/visit & 0.18 & 21.22 \\
\hline Nurse practitioner & 113.74/visit & 0.29 & 32.50 \\
\hline \multicolumn{4}{|l|}{ Diagnostic procedures } \\
\hline Keratometry & 5.72/eye & 6.43 & 36.77 \\
\hline Endothelial cell count & 3.68/eye & 1.43 & 5.25 \\
\hline Emergency outpatient visit (20 minutes) & & & \\
\hline Corneal specialist & 145.55/visit & 0.07 & 10.40 \\
\hline Optometrist & 112.87/visit & 0.04 & 4.03 \\
\hline Resident & 118.83/visit & 0.04 & 4.24 \\
\hline Consultation by telephone (10 minutes) & & & \\
\hline Corneal specialist & 19.53/call & 0.07 & 1.40 \\
\hline Optometrist & 3.12/call & 0.04 & 0.11 \\
\hline No shows & 3.12/visit & 0.14 & 0.45 \\
\hline Postoperative costs & & & $522.20 \pm 164.71$ \\
\hline Total costs per cross-linking treatment $(€)$ & Total \pm SD & & $1,754.06 \pm 177.23$ \\
\hline
\end{tabular}

